\begin{tabular}{|c|c|}
\hline \multirow{3}{*}{ 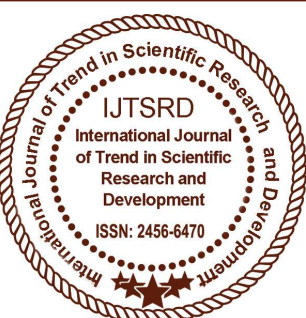 } & $\begin{array}{l}\text { International Journal of Trend in Scientific } \\
\text { Research and Development (IJTSRD) }\end{array}$ \\
\hline & International Open Access Journal \\
\hline & ISSN No: 2456 - 6470 | www.ijtsrd.com | Volume - 2 | Issue - 5 \\
\hline
\end{tabular}

\title{
A Study on the Influence of Stress on Psychological Wellbeing of BMTC Conductors
}

\author{
Roseline Florence Gomes ${ }^{1}$, Maria Reena ${ }^{2}$ \\ ${ }^{1}$ Lecturer, ${ }^{2}$ Special Educator \\ ${ }^{1}$ Department of Psychology, Jyoti Nivas College Autonomous, ${ }^{2}$ Learning Arc, \\ Bangalore, Karnataka, India
}

\begin{abstract}
Stress is conceptualized as any physical and psychological event that is perceived as potentially constituting physical harm or emotional distress. It's preoccupation with the negative emotion following the event (Roger, 1998). Psychological wellbeing highlights the core aspects of building positive relationships with others, personal mastery, independence, feeling of purpose and meaning of life. Bangalore Metropolitan Transport /n Cooperation (BMTC) is a government agency that operates the public bus service bus transport in Bangalore. The present study aims to explore the influences of stress on the psychological wellbeing of BMTC conductors.
\end{abstract}

The objectives were to examine the contextual relationship and effects of stress on the personal growth of conductors. The research will also provide information on how stress will influence psychological wellbeing of the BMTC conductors. Studies show the effect of job strain on the drivers performance (Usche, et.al.2016). The data was collected using the HSE's Management Standards Indicator Tool and psychological well being PWBQ (Carl Ryff 1989) on the selected sample. Results indicate that there is no influence of stress on psychological well being among conductors; the experimental group did not show significant increase or decrease in Psychological wellbeing after intervention.

Keywords: Psychological wellbeing, Stress, BMTC Conductors.

\subsection{INTRODUCTION}

Stress is reacting to hazard or challenges, or stress is the reaction to events that intimidate or challenge a person. It affects our psychological well-being. In accumulation to exceptional values and ideologies apprehended by people within each culture, there are distinctive sets of stressors or demands on individuals (Laungani, 1993). The ways in which individuals respond to these stressors are very culture specific. In other words, culture might impact the perceptions of stressors and development of strains (Bhagat, O’Driscoll, babakus etal., 1994; Keinana \& Perlberg, 1987). A moderator of stress, that may be culturedependent, is likely to be our social support (Behr and McGrath, 1992).

Psychological well-being reflects the speculation and measurement scales designed and advocated primarily by Carol Ryff. In her seminal paper, "Happiness is everything, or is it? Explorations on the meaning of psychological well-being", she contrasts this with subjective well-being or hedonic well-being. Ryff attempted to collaborate diverse conceptions of well-being from the ancient Greek to the modern psychological perspectives such as theories of Individuation from Carl Jung, Selfactualization from Abraham Maslow and others.

The term psychological well being (PWB) appears very simple and translucent but connotes to an extensive range of meanings frequently coupled with wellness. This concept has been comprehensively researched and the past six decades have provided innumerable theoretical requisites which refers to the PWB and its attributes are, flexibility and adaptability (Jung) 1939), fully functioning person (Rogers, 1961), 
employee factors (Maslow 1962), control on one's mind and body (Wilber, 1970) dying to a finite ego (Goleman, 1976) sense of meaning companion and selfless service, increased depth in intimate relationships, self- importance etc. Consequently these have generated an immense deal of research literature dealing with the issue of well being but no study seems to provide a comprehensive view of PWB. It was a work of Bradburn (1969) when the term of PWB first came in to use. He conducted an extensive social survey involving representatives of different demographic features in order to study the structure of PWB in 1969. This was followed by another survey in America by Campbell (1976) to identify the social indicators of well being and the Americans perception of 'Quality of life' Recently, Carol Ryff conducted a survey of samples Representing different age groups, sex groups and marital status and other Variables in 1995 with a view to identify the various dimension of PWB.

\subsection{Analytical Framework of the Study}

Surendra Dablholkar, Sehrish Khatib, Tejashree Dabholkar (2012) highlighted the aspects to identify psychological issues in navi mumbai bus conductors. The study investigated the fact that Mumbai bus conductors faced psychological problems such as stress, anxiety, depression and work place stress.

The effects of social support, job control, participative decision making practices, and locus of control upon the relationship between occupational stress and psychological well-being was greatly analyzed. The study discovered that complex interactions between stressors, locus of control, and social support or job autonomy in predicting psychological wellbeing (Kevin Daniels, Andrew Guppy 2011).

Work demands and stress in repetitive blue-collar work (Macdonald, Wendy 2003) was documented and evaluated to assess the formal and informal methods that was used to establish standard work rates or production targets within a sample of Australian work organizations. It focused predominantly on recruits performing repetitive work responsibilities, mostly in manufacturing industry. The project documented employee's evaluations of their required work rates and their levels of affective well-being and related these to various characteristics of work: the factors "pacing" task performance; the nature and level of task difficulty; the level of employees' perceived control or participation in decision-making; and the quality of job design factors related to job satisfaction

\subsection{Methodology}

Aim: To study the influence of stress on psychological wellbeing of bus conductors.

Hypothesis: There is no relationship between stress and psychological well-being among BMTC conductors

Purpose and Objectives: The purpose of this study is to examine the influence of stress on psychological wellbeing of BMTC Conductors. Specifically, the following research questions are assisted to determine the influence of Stress level among BMTC conductors and to establish the influence of Psychological wellbeing level among BMTC conductors.

Variables: Psychological well-being and Stress

\section{Operational definition:}

Psychological well-being: In the present study deals with satisfaction, self-esteem, positive effect, daily activities, life satisfaction, suicidal ideas, personal control and general wellness and efficacy.

Stress: Stress in the present study refers to the stress caused by one's job, feeling of tension, both at physical and emotion.

\section{Description of the Tools:}

Health Safety Executive (Health \& Safety at Work, 1974): It has 35 items in the stress scale constructed to measure the dimensions of demand, control, support, relationship; role and change; reliability and validity are measured using the scoring sheets.

\section{Scales of Psychological Well-Being (Ryff, 1989):}

There are items for six 14-item scales of psychological well-being constructed to measure the dimensions of autonomy, environmental mastery, personal growth, positive relations with others, purpose in life, and self-acceptance.

\subsection{Research Design}

The selection of the sample was through simple random sampling method. Then administration of HSE and Psychological wellbeing scale questionnaire by Carl Ryff (2012) to the conductors $(\mathrm{n}=60)$ aged 2535 years. The scores then calculated using the appropriate scoring norms and a correlation is used to 
verify the hypothesis. The sample size is $\mathrm{n}=60$ conductors, aged 25 - 35 years.

\subsection{Procedure}

The participants were first asked if he/she is ready to take up the test, the subject is assured confidentiality. The participant is provided with the questionnaires, HSE scale and PWQ questionnaire. The instructions are read carefully to the participant on the test before answering the questions, and clarify doubts if any beforehand. There is no time limit however both the scales should not take more than one hour. When the participants are ready and instructions were clearly understood, the questions are translated in Kannada to the participants. The responses were collected for both the scales. The data is collected from BMTC Conductors aged between 25-35 years. The size of the sample was 60,38 men and 22 women. And also an overall analysis of the influence of stress on psychological wellbeing on BMTC conductors is carried out. Data was analyzed using descriptive statistics and Pearson's correlation coefficient.

\subsection{Analysis and Interpretation}

The aim of the current study was to find if stress influences psychological wellbeing of I"BMTC conductors. The sampling technique that was used was simple random sampling technique was used to compare the values of the means from samples and to test the significance. The objective of the study the relationship between stress and psychological wellbeing among BMTC conductors.

On the sub scale of demand the conductors have an overall mean score of .93 , with standard deviation of .88. The result shows they have to work very fast and have achievable deadlines. The mean score is low under demand because the conductors have target of 8000 every month, which is not possible always to achieve the target due to various reasons such as metro, own transports etc. On the sub scale of control the conductors have an overall mean score of 1.85 with standard deviation of .784, which states that they can decide when to take break and have choice in deciding what work they want to do, mean score being low states that they cannot take break as they need to punch on time and more over traffic being hectic they cannot think even about food. On the sub scale of support the conductors have overall mean score 2.78 and standard deviation of .789 which states that they can rely on their head or superior to help them out with work problem and colleagues are willing to listen to their work related problems, sometimes they do not raise their voice as they scared to lose their jobs.

On the sub scale of relationship the conductors have overall mean core of 2.03 and standard deviation of .830 states that there is no anger or friction between colleagues and receive respect at work from their colleagues, family. Mean having low score in relationship says they cannot spend maximum time with spouse and children as they do not have long holidays.

On the sub scale of role the conductors have overall mean score of 3.22 and standard deviation of 1.018 which states that they are clear what is expected from them at work, low mean states they find difficult to reach their target even after knowing it is compulsory to achieve their deadlines.

On the sub scale of change the conductors have overall mean score of 3.41 and standard deviation of 1.069 which states that staffs are always consulted about change at work, mean score being low indicates sometimes if other staff are on leave they still need to work and work even on their off and take leave on other days.

On the sub scale of demand the conductors have an overall mean score of .93, with standard deviation of .88. The result shows they have to work very fast and have achievable deadlines. The mean score is low under demand because the conductors have target of 8000 every month, which is not possible always to achieve the target due to various reasons such as metro, own transports etc.

On the sub scale of control the conductors have an overall mean score of 1.85 with standard deviation of .784, which states that they can decide when to take break and have choice in deciding what work they want to do, mean score being low states that they cannot take break as they need to punch on time and more over traffic being hectic they cannot think even about food.

On the sub scale of support the conductors have overall mean score 2.78 and standard deviation of .789 which states that they can rely on their head or superior to help them out with work problem and colleagues are willing to listen to their work related 
problems, sometimes they do not raise their voice as they scared to lose their jobs.

On the sub scale of relationship the conductors have overall mean core of 2.03 and standard deviation of .830 states that there is no anger or friction between colleagues and receive respect at work from their colleagues, family. Mean having low score in relationship says they cannot spend maximum time with spouse and children as they do not have long holidays.

On the sub scale of role the conductors have overall mean score of 3.22 and standard deviation of 1.018 which states that they are clear what is expected from them at work, low mean states they find difficult to reach their target even after knowing it is compulsory to achieve their deadlines.

On the sub scale of change the conductors have overall mean score of 3.41 and standard deviation of 1.069 which states that staffs are always consulted about change at work, mean score being low indicates sometimes if other staff are on leave they still need to work and work even on their off and take leave on other days.

On the sub scale of autonomy the conductors have an overall mean score of 53.07, with standard deviation of 10.00. The result shows participant is not afraid to raise his/her opinions, even when they are in opposition to the opinions of most people. Therefore the results indicate that the participants are selfdetermining and independent; able to resist social pressures to think and act in certain ways; regulates behavior from within; evaluates self by personal standards.

On the sub scale of environmental mastery the conductors have an overall mean score of 49.49 with standard deviation of 9.511, which states that they feel that they are quite good in managing many responsibilities of their daily life. Therefore the results indicate that the participants has a sense of mastery and competence in managing the environment; controls complex array of external activities; makes effective use of surrounding opportunities; able to choose or create contexts suitable to personal needs and values.

On the sub scale of personal growth the conductors have overall mean score 54.49 and standard deviation of 11.094 which states that participants feel that they continue to learn more about themselves as times go by. Therefore the results indicate that the participants has a feeling of continued development; sees self as growing and expanding; is open to new experiences; has sense of realizing his or her potential; sees improvement in self and behavior over time; is changing in ways that reflect more self knowledge and effectiveness. On the sub scale of positive relationship the conductors have overall mean core of 52.41 and standard deviation of 10.393 states that with others they sympathize with each other's problems. Therefore the results indicate that the participants has warm satisfying, trusting relationships with others; is concerned about the welfare of others; capable of strong empathy, affection, and intimacy; understands give and take of human relationships. On the sub scale of purpose in life the conductors have overall mean score of 50.54 and standard deviation of 8.856 which states how participant feel satisfied and pleased with how things have turned in their life. Therefore the results indicate that the participants has goals in life and a sense of directedness; feels there is meaning to present and past life; holds beliefs that give life purpose; has aims and objectives for living. On the sub scale of self acceptance the conductors have overall mean score of 50.42 and standard deviation of 8.937 which states that participant in general feels confident and positive about themselves. Therefore the results indicate that the participants Possesses a positive attitude toward the self; acknowledges and accepts multiple aspects of self including good and bad qualities; feels positive about past life.

\subsection{Summary and Conclusion}

The aim of the current study was to find if stress influences psychological wellbeing of BMTC conductors. The objective of the study is to compare the relationship between stress on psychological wellbeing among BMTC conductors, among which 22 were women and 38 were men. 60 samples were chosen between age group from 25 to 35 . The samples reject the hypothesis which states that Influence of stress on psychological wellbeing on BMTC conductors.

The study of sample was taken from BMTC which only specific depot was assigned. BMTC has overall name and good management as the management varies from place to place, if study was done on other conductors overall then it must have proved 
hypothesis, as most participants were feeling discomfort where they would lose their job and were surrounded by other colleagues.

\section{Bibliography}

1. Abhishek Singh, M. C. (2013). A descriptive study of perceived stress among the North Indian nursing undergraduate students.

2. Altindis, H. O. (2011). Big five personality factors and other elements in understanding work stress of Turkish health care professionals". African Journal of Business Management, 10462-10473.

3. Anthikad, J. (2007). Psychology for Graduate Nurses. New Delhi. : Jaypee Brothers Medical Publishers Pvt. Ltd, .

4. Dunham, G. E. (2005). Perceived Stress and Health-Promoting Behaviors in Nursing Students Using Pender's Health Promotion Model. UK: Masters Theses. Paper 228.

5. Ebstrup J F, E. L. (2011). Association between the Five Factor personality traits and perceived stress: is the effect mediated by general self-efficacy?" Denmark: Pub Med - indexed for MEDLINE.

6. Ecie, M. T. (2013). Relationships among nursing burnout, the big five personality factors, and overall self-concept: the impact of assessing common method variance. Tennessee.
7. Gardiner, K. (2006). The Effects of personality and perceived stress on the well being of nursing and psychology students: a cross-sectional study. uk: Psychology Undergraduate thesis collection.

8. Janjhua, Y. \&. (2012). Behavior of Personality Type toward Stress and Job Performance: A Study of Healthcare Professionals. . Journal of Family Medicine and Primary Care, 109-113.

9. Martin, B. (2014). 2013. Stress and Personality, 504-98.

10. McLeod, S. A. (2010). Stress Response. Proceedings of the Royal Society of Medicine, 4850.

11. Phillips, D. A. ((2013). Perceived Stress. springer.

12. Picket, A. E. (2014). A Correlational Study between Hardiness and the. Boiling Springs, North Carolina: ProQuest LLC.

13. Rashaun Roberts, P. L. (2012.). Alleviating Job Stress in Nurses. Medscape, 211-18.

14. Rashaun Roberts, P. L. (2012). Alleviating Job Stress in Nurses. Medscape.

15. Society, T. A. (2012). Understanding and managing stress. Melbourne: The Australian Psychological Society Limited. 